\title{
Analysis of inelastic pion-nucleus scattering within the microscopic optical potential and the in-medium effect on $\pi N$ amplitude in nuclei
}

\author{
V.K.Lukyanov*, E.V.Zemlyanaya, K.V.Lukyanov, \\ Joint Institute for Nuclear Research, 141980 Dubna, Russia \\ E-mail: vlukyanov@jinr.ru
}

\section{I.A.M. Abdul-Magead}

Cairo University, Cairo, Giza, Egypt

E-mail: iabudanckash@gmail.com

\begin{abstract}
The microscopic model of optical potential (OP) is applied for calculations of the $\pi^{ \pm}$inelastic scattering on the nuclei ${ }^{28} \mathrm{Si},{ }^{58} \mathrm{Ni},{ }^{40} \mathrm{Ca},{ }^{208} \mathrm{~Pb}$ at energies $160,180,230$ and $290 \mathrm{MeV}$ with excitations of the $2^{+}$and $3^{-}$collective states. In so doing we use the nuclear density distributions and the parameters of the $\pi \mathrm{N}$-scattering amplitudes earlier obtained by fitting the pion-nucleus elastic cross sections. Thus for inelastic scattering, the only adjusted parameters are the quadrupole $\beta_{2}$ and octupole $\beta_{3}$ deformations of nuclei. The cross sections were obtained by solving the relativistic wave equation, and thus the relativistic and distortion effects in initial and final channels of the process were accounted for exactly. The calculated cross sections were found to be in a fairly well agreement with the corresponding experimental data. One should underline an important role of the nuclear in-medium effect on the $\pi \mathrm{N}$-scattering amplitude that reveals itself in the both elastic and inelastic scattering.
\end{abstract}

XXII International Baldin Seminar on High Energy Physics Problems, September 15-20, 2014

JINR, Dubna, Russia

${ }^{*}$ Speaker. 


\section{Introduction}

In analysis of the data on inelastic scattering of pions from nuclei we use the microscopic folding-like optical potential (OP) derived in [1] for elastic scattering. This OP has been successfully applied for elastic scattering of the K- and pi-mesons on nuclei [2], [3], [4], [5]. It is constructed as a folding integral of a nuclear density form factor and the pion-nucleon amplitude of scattering. When using this OP at intermediate energies we avoid problems inherent in the models (see, e.g., [6]) based on the Glauber high-energy approximation for the scattering amplitudey [7]. Instead, for calculations of cross sections we apply the Klein-Gordon equation and thus take into account the relativistic and distortion effects exactly. Also, the folding-like OP is presented in a generalized form by introducing the small axiallity of the distance vector $\mathbf{r}$ of motions, and thus one can obtain the respective optical potential $U\left(\mathbf{r},\left\{\alpha_{\lambda \mu}\right\}\right)$ depended on the deformation variables $\alpha_{\lambda \mu}$ inherent in the collective motion of a nucleus. This enables one to consider the inelastic cross sections with excitations of the low lying $2^{+}$and $3^{-}$collective states of nuclei. The object of this paper is the detailed analysis of inelastic scattering of pions from nuclei at different energies in the region of the 33-resonance. It was shown in [5] that at these energies, the in-medium effects on the $\pi \mathrm{N}$ amplitude play an substantial role in the pion-nucleus elastic scattering. So, in this connection the question arises on the role of this effect in inelastic scattering of pions on the same nuclei and at the same energies when excitations of their low lying collective states take place.

In section 2, we present the main formulas for construction of the microscopic OP for elastic and inelastic scattering of pions. In section 3, calculations of the pion-nucleus inelastic cross sections are presented and the fitting parameters of the nuclear deformation and parameters of the $\pi N$ elementary amplitude are discussed.

\section{Basic formulas and calculations}

We start with the microscopic nuclear OP [1] given in the general form

$$
U(r)=-\frac{(\hbar c) \beta_{c}}{(2 \pi)^{2} k} \int e^{-i \mathbf{q r}} \rho(\mathbf{q}) F_{\pi N}(q) d^{3} q,
$$

where $\beta_{c}$ is the ratio of the c.m. pion velocity to the light one ${ }^{1}$. Below in calculations the pionnucleon amplitude of scattering is taken as follows

$$
F_{\pi N}(q)=\frac{k}{4 \pi} \sigma[i+\alpha] \cdot f(q), \quad f(q)=e^{-\beta q^{2} / 2},
$$

where $\beta$ is the slope parameter, $\sigma$ and $\alpha$ are the isospin averaged $\pi \mathrm{N}$ total cross section and the ratio of the real to imaginary part of the amplitude of scattering $F_{\pi N}(0)$ at forward angles, while $\rho(\mathbf{q})$ is the form factor of a density distribution function of bare nucleons in a nucleus normalized to atomic number $A$

$$
\rho(\mathbf{q})=\int e^{i \mathbf{q r}} \rho(\mathbf{r}) d^{3} r .
$$

\footnotetext{
${ }^{1}$ In eq.(2.1), it is used units $\mathrm{MeV}$ and fm, and then $\hbar c=197.327 \mathrm{MeV} \cdot \mathrm{fm}$. In the other cases we employ the natural system of units where $\hbar=c=1$, and thus $E, T, k, m$ have the same dimension [MeV].
} 


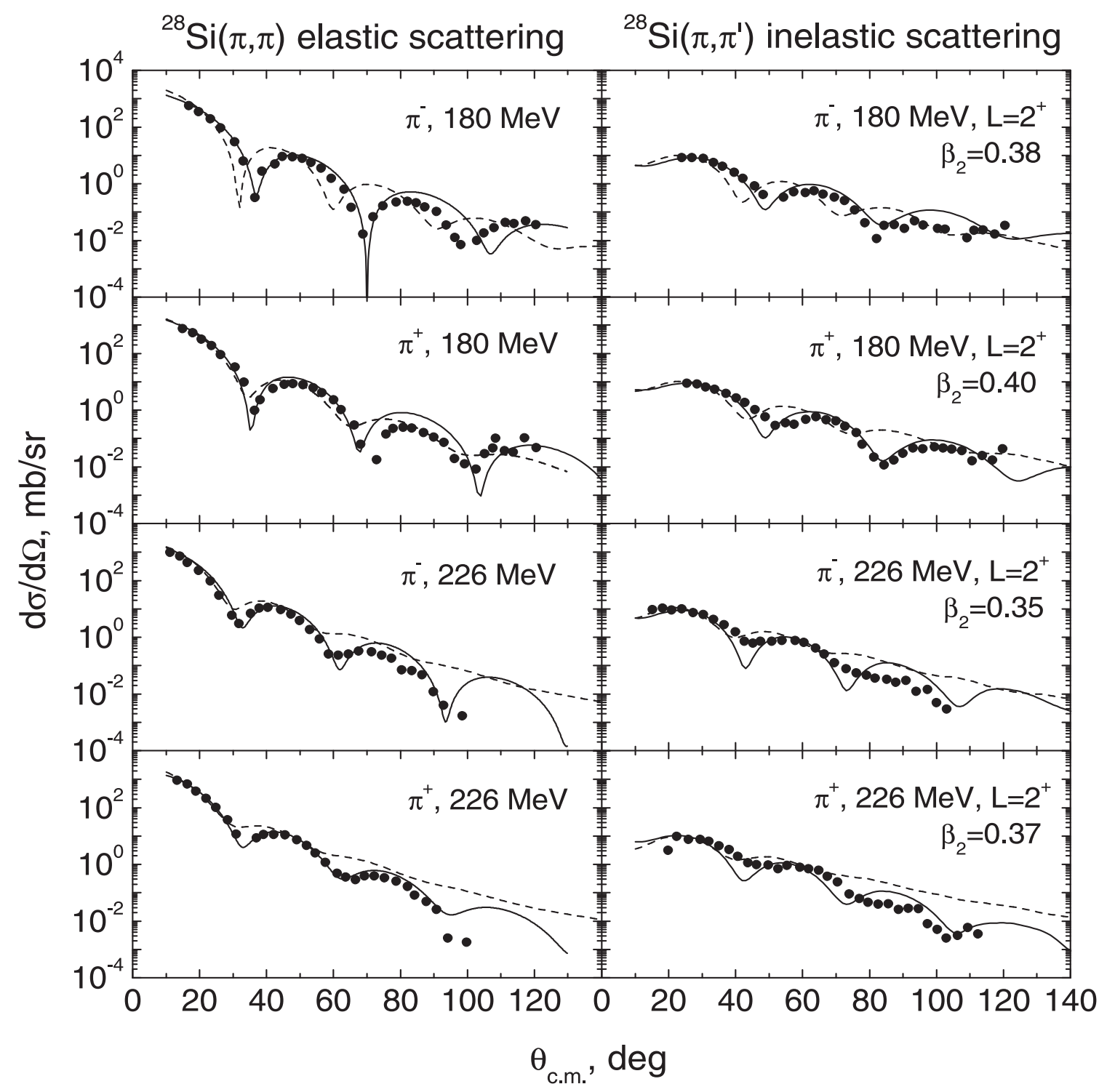

Figure 1. Comparisons of calculated differential cross sections of $\pi^{ \pm}$-mesons scattered on ${ }^{28} \mathrm{Si}$ at $T_{l a b}=180$ and $226 \mathrm{MeV}$ to experimental data from [13]. Left panel is for elastic scattering, right panel - for inelastic scattering with excitations of the respective $\lambda=2^{+}$and (or) $2^{-}$states. In both panels, dashed curves are calculations with parameters $\sigma, \alpha, \beta_{\pi}$ of the free $\pi N$ amplitudes at respective energies, solid lines are for those fitted in [5] to the data on elastic scattering. The values of the fitted deformation parameters are shown on the right panel.

Usually in calculations of the pion-nucleus elastic cross sections the nuclear densities are taken as a spherically-symmetric functions $\rho(\mathbf{r})=\rho(r)$. So, in our preceding study [5] of the pion scattering on a set of nuclei the nuclear densities $\rho(r)$ were taken in the form of the fermi functions with the known fixed parameters of the radius $R$ and diffuseness $a$. On the other hand, the parameters of the amplitude of scattering (2.2) of pions on the bounded nuclear nucleons were fitted by comparisons of the calculated pion-nucleus cross sections with existing experimental data, and thus the table of 


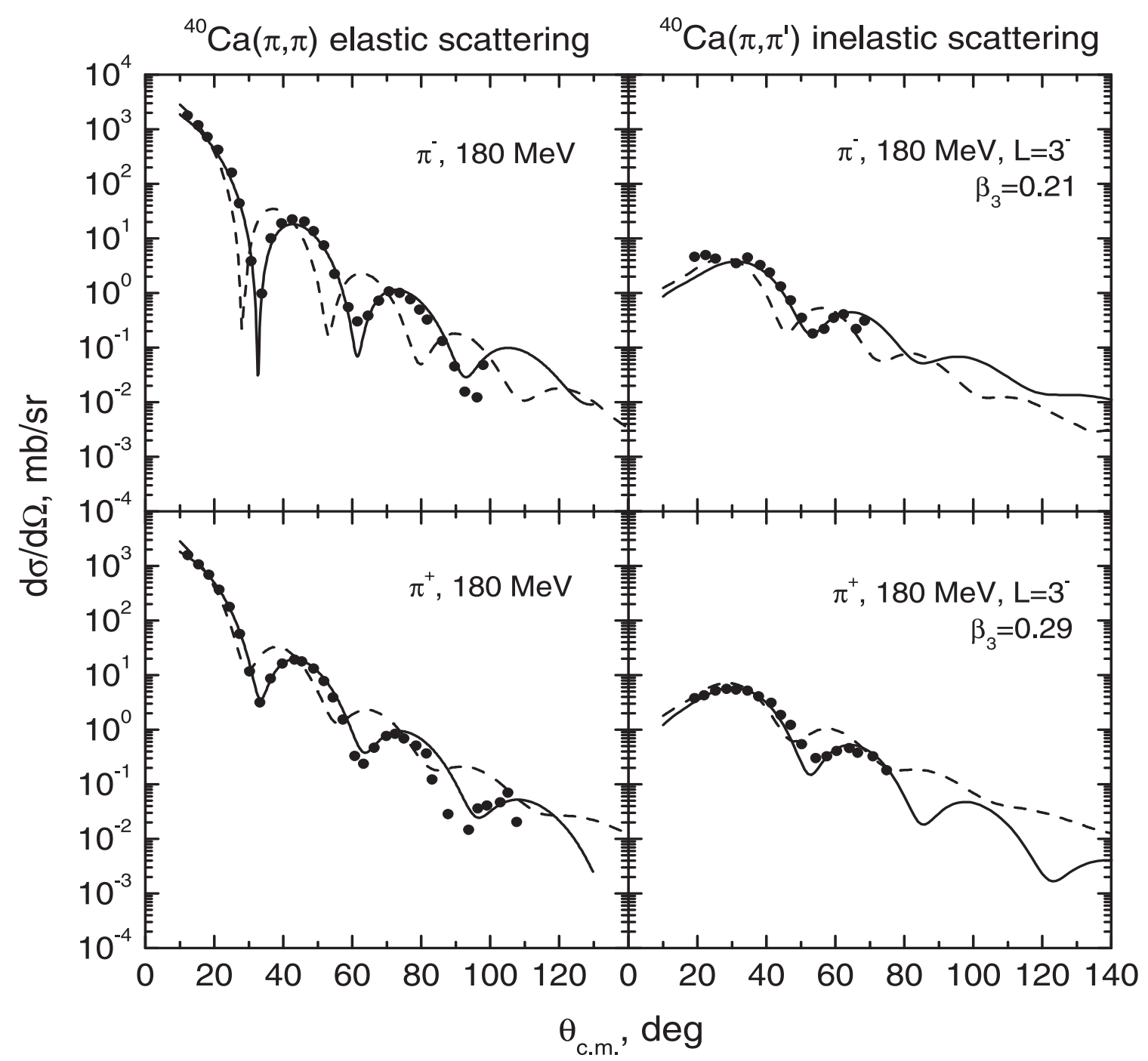

Figure 2. The same as in Fig. 1 but for the target nucleus ${ }^{40} \mathrm{Ca}$, the energy of pions $T_{\text {lab }}=180 \mathrm{MeV}$, and experimental data are from [14].

the so-called in-medium parameters $\sigma, \alpha, \beta$ were established at different collision energies. In fact, they occur rather different as compared to those obtained from scattering on free nucleons.

In the present study we intend to verify if these in-medium parameters would remain their values as before but for inelastic scattering of pions at the same energies on the same nuclei. To this end we construct the transition potentials for inelastic scattering starting from the standard prescription [8] to deform the $\mathbf{r}$-surfaces by adding a small axially-symmetric admixture

$$
\mathbf{r} \Rightarrow r+\delta^{(\lambda)}(\mathbf{r}), \quad \delta^{(\lambda)}(\mathbf{r})=-r(r / R)^{\lambda-2} \sum_{\mu} \alpha_{\lambda \mu} Y_{\lambda \mu}(\hat{r}), \quad \lambda=2,3
$$

Here $\alpha_{\lambda \mu}$ are the collective motion variables of a nucleus. 
Substituting (2.4) into (2.3) and then in (2.1), and terminating their expansions at linear terms in $\delta^{(\lambda)}(\mathbf{r})$ one obtains the respective density and its form factor

$$
\begin{gathered}
\rho(\mathbf{r})=\rho(r)+\rho_{\lambda}(r) \sum_{\mu} \alpha_{\lambda \mu} Y_{\lambda \mu}(\hat{r}), \quad \rho_{\lambda}(r)=-r \frac{d \rho(r)}{d r}(r / R)^{\lambda-2} \\
\rho(\mathbf{q})=\rho(q)+\rho_{\lambda}(q) i^{\lambda} \sum_{\mu} \alpha_{\lambda \mu} Y_{\lambda \mu}(\hat{q}),
\end{gathered}
$$

where

$$
\rho(q)=4 \pi \int j_{0}(q r) \rho(r) r^{2} d r, \quad \rho_{\lambda}(q)=4 \pi \int j_{\lambda}(q r) \rho_{\lambda}(r) r^{2} d r .
$$

Finally, substituting (2.6) in (2.1) we obtain the direct and transition potentials for calculations of elastic and inelastic scattering

$$
\begin{gathered}
U(\mathbf{r})=U(r)+U^{(\lambda)}(\mathbf{r}), \quad U^{(\lambda)}(\mathbf{r})=U_{\lambda}(r) \sum_{\mu} \alpha_{\lambda \mu} Y_{\lambda \mu}(\hat{r}), \\
U(r)=-\frac{\hbar v}{(2 \pi)^{2}} \sigma_{N N}\left(\alpha_{N N}+i\right) \int j_{0}(q r) \rho(q) f(q) q^{2} d q, \\
U_{\lambda}(r)=-\frac{\hbar v}{(2 \pi)^{2}} \sigma_{N N}\left(\alpha_{N N}+i\right) \int j_{\lambda}(q r) \rho_{\lambda}(q) f(q) q^{2} d q .
\end{gathered}
$$

In so way the obtained microscopic potential (2.9) should be inserted into the Klein-Gordon equation for the case of $E \gg U$ with the neglected terms of the order of $(U / E)^{2} \ll 1$ and thus presented in the form of the non-relativistic wave equation

$$
\left(\Delta+k^{2}\right) \psi(\mathbf{r})=2 \bar{\mu} \cdot\left[U(r)+U_{c}(r)\right] \psi(\mathbf{r}),
$$

where the relativistic velocity $\beta_{c}=v_{c . m .} / c=k_{l a b} /\left[E_{l a b}+m_{\pi}^{2} / M_{A}\right]$ is expressed through the total laboratory energy $E_{\text {lab }}=\left(k_{\text {lab }}^{2}+m_{\pi}^{2}\right)^{1 / 2}=T_{\text {lab }}+m_{\pi}$ and momentum $k_{\text {lab }}$ and $M_{A}$, the mass of a target nucleus. Here the spherically symmetric part of the Coulomb potential $U_{c}(r)$ is taken for the charged sphere with the radius $R_{c}=r_{c} A^{1 / 3}$, where $r_{c}=1.3 \mathrm{fm}$. Also, $k$ is the relativistic momentum in c.m. system $k=M_{A} \sqrt{T_{l a b}\left(T_{l a b}+2 m_{\pi}\right)} / \sqrt{\left(m_{\pi}+M_{A}\right)^{2}+2 M_{A} T_{l a b}}$ and the relativistic reduce mass $\bar{\mu}=\bar{m}_{\pi} M_{A} /\left(\bar{m}_{\pi}+M_{A}\right)$ with $\bar{m}_{\pi}=\sqrt{k^{2}+m_{\pi}^{2}}=T_{c . m}$. $+m_{\pi}$. Finally, the transformed wave equation (2.11)can be computed using the program DWUCK4 [9], and thus one accounts for automatically effects of relativization and distortions of the relative motion wave functions in the field of a target nucleus. In the program, the spherically symmetric part of the optical potential $U_{\text {opt }}(r)=U(r)+U_{c}(r)$ provides elastic scattering calculations while the $U^{(\lambda)}(\mathbf{r})+U_{c}^{(\lambda)}(\mathbf{r})$ is the transition OP used for calculations of inelastic scattering cross sections with excitations of the $2^{+}$ and $3^{-}$collective states of nuclei. In inelastic processes, the Coulomb interaction is incorporated in the DWUCK4 program in the form of eq.(2.8) but with the radial part

$$
U_{c, \lambda}(r)=\frac{3 Z_{A} Z_{\pi} e^{2}}{2 \lambda+1} \cdot \frac{R_{c}^{\lambda}}{r^{\lambda+1}}, \quad r>R_{c}
$$

that corresponds to the external multiple decomposition of the Coulomb potential for the uniformed charged sphere. In calculations, the amplitude of inelastic scattering is taken in the framework of 


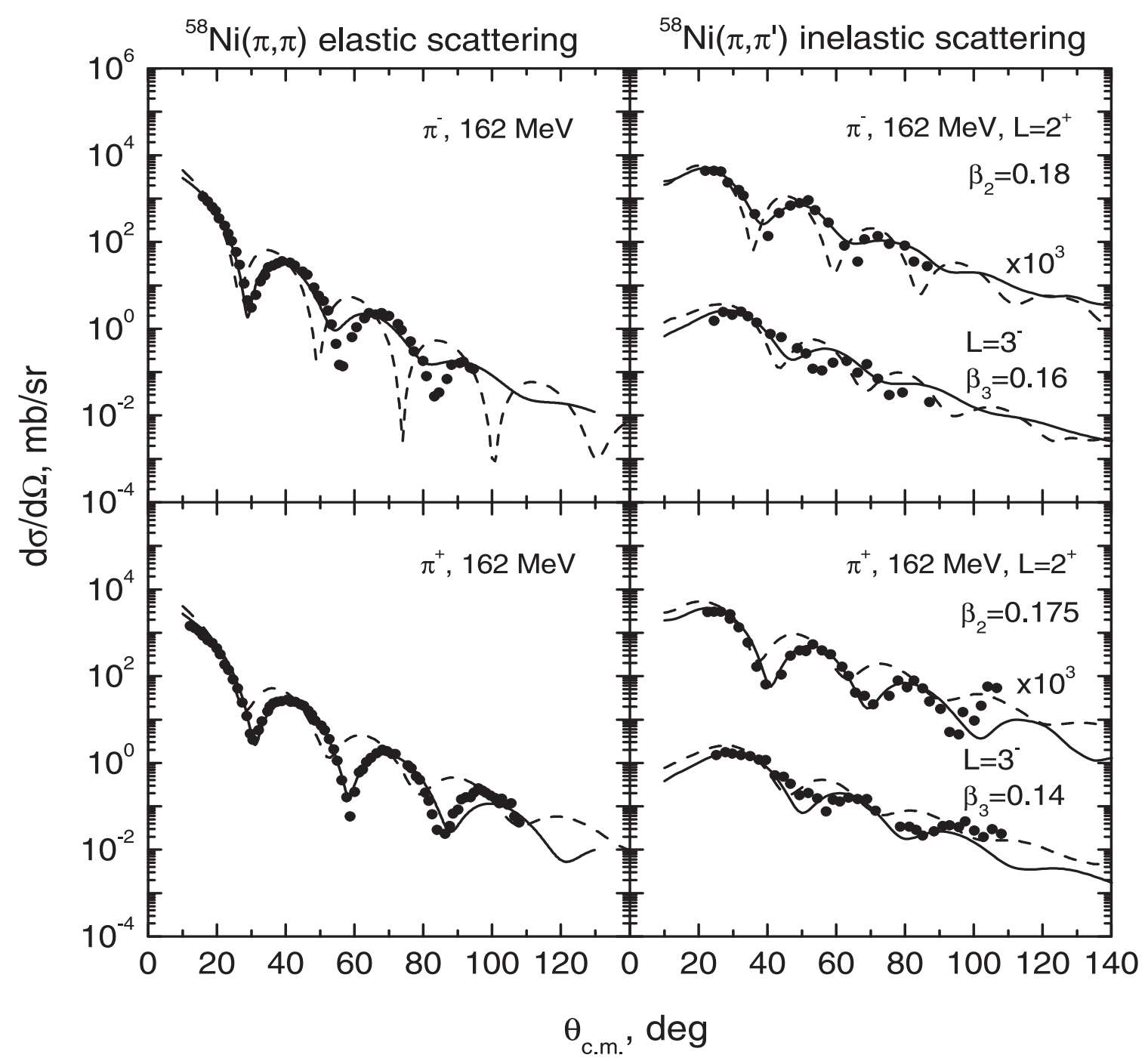

Figure 3. The same as in Fig. 1 but for the target nucleus ${ }^{58} \mathrm{Ni}$, the energy of pions $T_{\text {lab }}=162 \mathrm{MeV}$, and experimental data are from [15].

the distorted wave Born approximation (DWBA) where the respective matrix element has a linear dependence on the transition potential while the distorted waves in initial and final channels $\psi^{( \pm)}(\mathbf{r})$ are calculated using the $U_{\text {opt }}(r)=U(r)+U_{c}(r)$ potential, and thus

$$
T_{B A}^{\lambda}=\sum_{\mu}<B\left|\alpha_{\lambda \mu}\right| A>\int \psi_{b}^{(-) *}\left(\mathbf{r}, \mathbf{k}_{b}\right)\left[U_{\lambda}(r)+U_{c, \lambda}(r)\right] Y_{\lambda \mu}(\hat{r}) \psi_{a}^{(+)}\left(\mathbf{r}, \mathbf{k}_{a}\right) d \mathbf{r}_{a} d \mathbf{r}_{b}
$$

Here the structure matrix element $\left\langle B\left|\alpha_{\lambda \mu}\right| A>\right.$ provides the $(\lambda \mu)$-transition from the ground to excited state of a nucleus. In the case of the even-even nuclei one has $|A>=| I_{A}, M_{A}>\left(I_{A}=M_{A}=\right.$ $0),<B\left|=<I_{B}, M_{B}\right|\left(I_{A}=\lambda, M_{A}=\mu\right)$, and the transition matrix element is [8]

$$
\begin{aligned}
&<B\left|\alpha_{\lambda \mu}\right| A>=\left(I_{A} \lambda M_{A} \mu \mid I_{B} M_{B}\right)<I_{B}|| \alpha_{\lambda 0}|| I_{A}>= \\
&=(0 \lambda 0 \mu \mid \lambda \mu)<\lambda|| \alpha_{\lambda 0}|| 0>=(1 / \sqrt{2 \lambda+1}) \beta_{\lambda}
\end{aligned}
$$




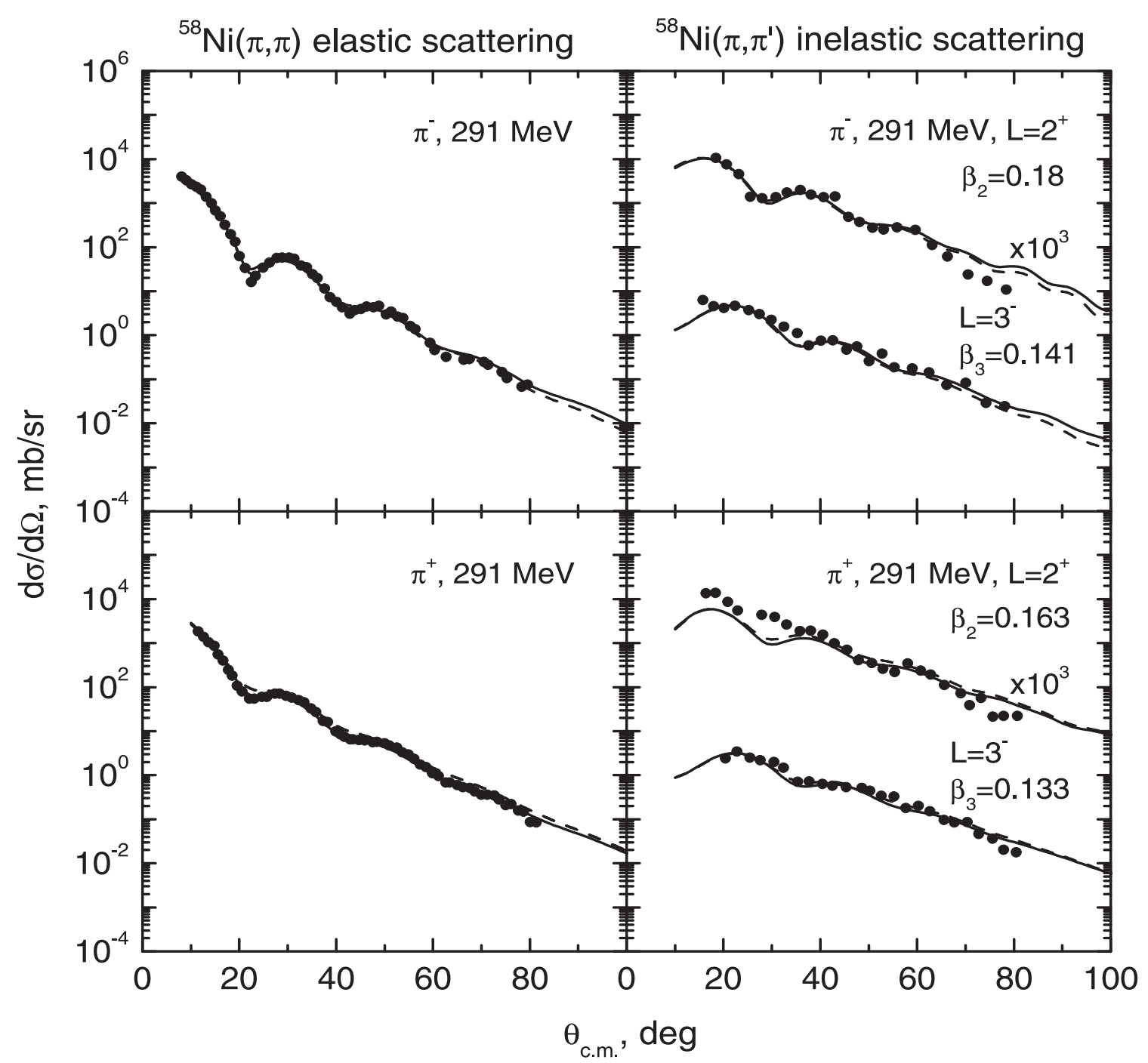

Figure 4. The same as in Fig. 1 but for the target nucleus ${ }^{58} \mathrm{Ni}$, the energy of pions $T_{\text {lab }}=291 \mathrm{MeV}$, and experimental data are from [16].

where $\beta_{\lambda}(\lambda=2,3)$ is a deformation parameter to be fitted in our study.

\section{Results and discussion}

In Figs.1-5 we present the experimental and calculated differential elastic and inelastic cross sections for scattering of $\pi^{ \pm}$-mesons on the even-even nuclei ${ }^{28} \mathrm{Si},{ }^{40} \mathrm{Ca},{ }^{58} \mathrm{Ni}$, and ${ }^{208} \mathrm{~Pb}$ at energies $T_{l a b}=162,180,226$ and $291 \mathrm{MeV}$ with excitations of the low-lying $2^{+}$and $3^{-}$collective states. In calculations of inelastic scattering we take the same nuclear fermi density parameters for the radius 


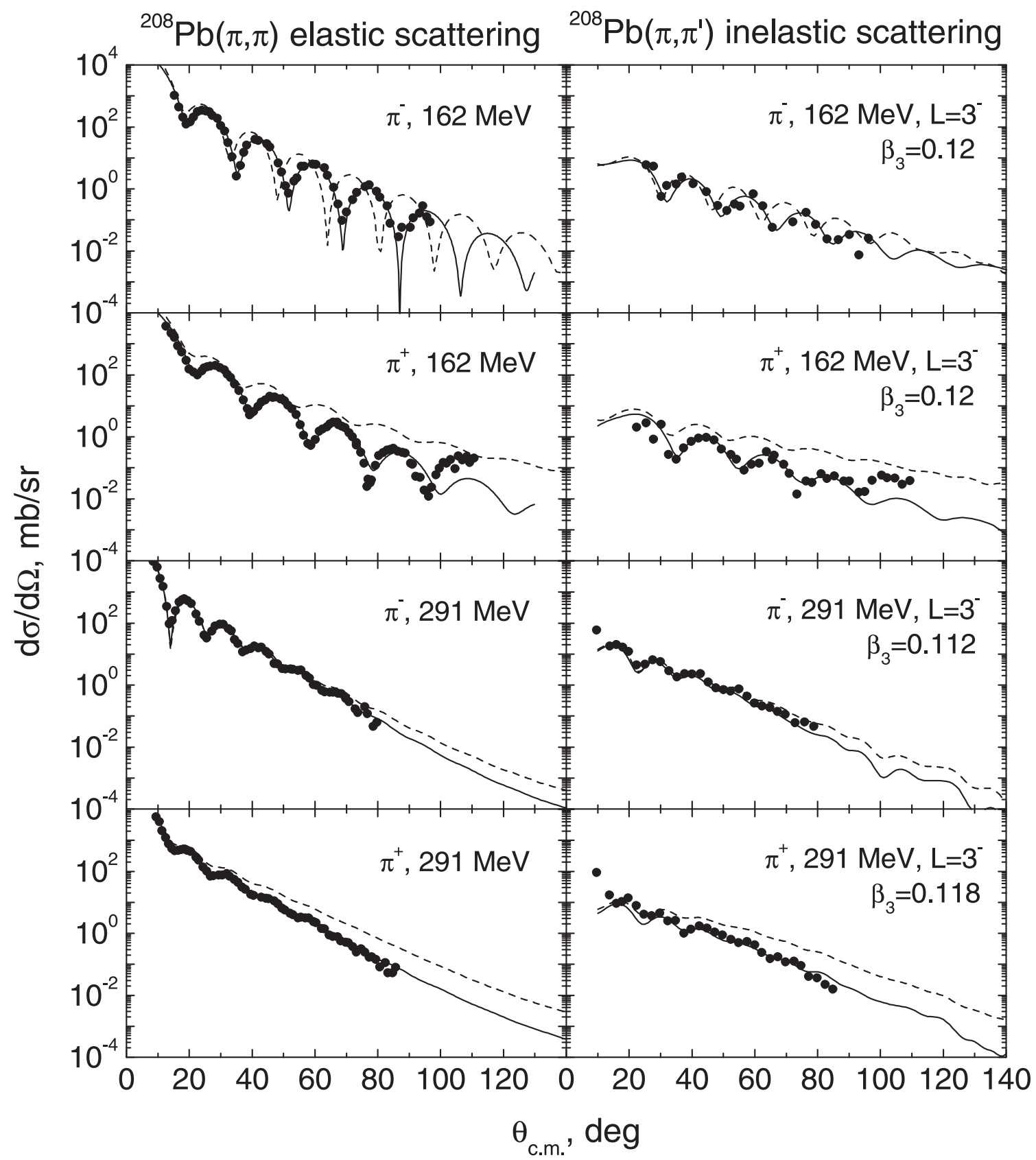

Figure 5. The same as in Fig. 1 but for the target nucleus ${ }^{208} \mathrm{~Pb}$, the energy of pions $T_{l a b}=162$ and $291 \mathrm{MeV}$, and experimental data are from [16].

$R$ and diffuseness $a$ as they were used for the $\pi$ A elastic scattering in [5]. Their values are 3.134 and 0.477 for ${ }^{28} \mathrm{Si}$ [10]; 3.593 and 0.493 for ${ }^{40} \mathrm{Ca}$ [10]; 4.08 and 0.515 for ${ }^{58} \mathrm{Ni}$ [11]; 6.654 and 0.475 for ${ }^{208} \mathrm{~Pb}$ [12]. As to the in-medium parameters $\sigma, \alpha, \beta$ of the pion-nucleon amplitude (2.2), they were fitted in [5] when reproducing the respective experimental $\pi^{ \pm} A$ data from [13], [14], [15], [16] on the elastic scattering of pions on the same nuclei at the same energies (see Table 1).

On the left panels of all the Figures we exhibit the curves for the differential elastic scattering and on the right panel - the respective inelastic scattering cross sections. For each nucleus, the 
Table 1: The best-fit in-medium parameters $\sigma, \alpha, \beta$ and the free one $\sigma_{0}, \alpha_{0}, \beta_{0}$ for the $\pi \mathrm{N}$ elastic scattering amplitude, and the adjusted deformation parameters $\beta_{2,3}$ for inelastic cross sections.

\begin{tabular}{|c|c||c|c|c||c|c|c||c|c|}
\hline reaction & $T_{\text {lab }}, \mathrm{Mev}$ & $\sigma, \mathrm{fm}^{2}$ & $\alpha$ & $\beta, \mathrm{fm}^{2}$ & $\sigma_{0}, \mathrm{fm}^{2}$ & $\alpha_{0}$ & $\beta_{0}, \mathrm{fm}^{2}$ & $\beta_{2}$ & $\beta_{3}$ \\
\hline$\pi^{-}+{ }^{58} \mathrm{Ni}$ & 162 & 10.95 & -0.16 & 1.10 & 12.135 & 0.369 & 1.065 & 0.18 & 0.16 \\
$\pi^{+}+{ }^{58} \mathrm{Ni}$ & & 8.86 & 0.44 & 0.81 & & & & 0.175 & 0.14 \\
$\pi^{-}+{ }^{208} \mathrm{~Pb}$ & & 9.69 & 0.34 & 1.02 & & & & & 0.12 \\
$\pi^{+}+{ }^{208} \mathrm{~Pb}$ & & 6.18 & 0.58 & 1.24 & & & & & 0.12 \\
\hline$\pi^{-}+{ }^{28} \mathrm{Si}$ & 180 & 9.33 & 0.43 & 0.28 & 12.76 & 0.114 & 0.994 & 0.38 & \\
$\pi^{+}+{ }^{28} \mathrm{Si}$ & & 7.75 & 0.76 & 0.49 & & & & 0.40 & \\
$\pi^{-}+{ }^{40} \mathrm{Ca}$ & & 9.65 & 0.23 & 0.28 & & & & & 0.21 \\
$\pi^{+}+{ }^{40} \mathrm{Ca}$ & & 5.75 & 1.09 & 0.69 & & & & & 0.29 \\
\hline$\pi^{-}+{ }^{28} \mathrm{Si}$ & 226 & 7.43 & 0.6 & 0.167 & 9.15 & -0.515 & 0.698 & 0.35 & \\
$\pi^{+}+{ }^{28} \mathrm{Si}$ & & 5.87 & 1.08 & 0.42 & & & & 0.37 & \\
\hline$\pi^{-}+{ }^{58} \mathrm{Ni}$ & 291 & 4.79 & -0.85 & 0.279 & 4.76 & -0.95 & 0.434 & 0.18 & 0.141 \\
$\pi^{+}+{ }^{58} \mathrm{Ni}$ & & 5.58 & -0.66 & 0.354 & & & & 0.163 & 0.133 \\
$\pi^{-}+{ }^{208} \mathrm{~Pb}$ & & 4.47 & -1.07 & 0.672 & & & & & 0.112 \\
$\pi^{+}+{ }^{208} \mathrm{~Pb}$ & & 5.52 & -0.46 & 0.581 & & & & & 0.118 \\
\hline
\end{tabular}

solid curve for elastic scattering is the fitted cross section from [5], and the corresponding solid curve for inelastic scattering is that calculated using the same fitted in-medium parameters $\sigma, \alpha$, $\beta$ from elastic scattering while the only parameter adjusted to inelastic scattering is the $\beta_{2}$ (or $\beta_{3}$ ) deformation for the quadruple (or octuple) excitation of a nucleus. For comparisons we show also the dashed curves calculated by using the "free parameters" for the $\pi \mathrm{N}$-amplitude of scattering on free nucleons as they done in [17] and [3]. It is seen, first, that in all the cases we obtain the fairly well agreement with experimental data for inelastic scattering and that we need not to vary the $\pi \mathrm{N}$ in-medium parameters responsible to elastic scattering cross sections. Instead, when we take the "free parameters" then one gets the significant disagreements with the data for both elastic and inelastic cross sections. Then, this in-medium effect reveals itself especially at energies in the region of 33-resonance with its maximum at $180 \mathrm{MeV}$ while at its boundary energy about $291 \mathrm{MeV}$ the in-medium effect becomes rather weak as is seen in Figs.4,5 where the difference between solid and dash curves is not too large with an exception the $\pi^{+208} \mathrm{~Pb}$ scattering. As to the obtained values of the nuclear deformation parameters, for a definite nucleus they occur almost independent of the energy of scattered pions $\pi^{+}$or $\pi^{-}$, i.e. they are seem to be the really structure parameters.

The work was partly supported by RFBR (under grant No.13-01-00060a) and the JINR-Egypt Cooperation Program.

\section{References}

[1] Lukyanov V.K., Zemlyanaya E.V., Lukyanov K.V. Nucleus-nucleus scattering in the high-energy approximation and optical folding potential. Phys. Atom. Nucl. 69 (2006) no.2, 240; Preprint JINR, P4-2004-114, Dubna (2004) 
[2] Lukyanov V.K., Zemlyanaya E.V., Lukyanov K.V., Hanna K.M. Microscopic $K^{+}$-nucleus optical potential and calculations of differential elastic scattering cross sections and total reaction cross sections. Phys. Atom. Nucl. 73 (2010) no.8, 1443

[3] Lukyanov V.K., Zemlyanaya E.V., Lukyanov K.V., Ali El Lithy, Ibrahim Abdulmagead, Slowinski B. Analisis of elastic scattering of pi-mesons by nuclei within the microscopic optical potential. Bull. Russ. Acad. Sciences, Physics, 77 (2013) no.4, 427

[4] Lukyanov V.K., Zemlyanaya E.V, Lukyanov K.V., Ali El Lithy, Ibrahim Abdulmagead Analisis of inelastic scattering of $\pi$-mesons from nuclei within the microscopic optical potential. Bull. Russ. Acad. Sciences, Physics. 78 (2014) no.5, 421

[5] Lukyanov V.K., Zemlyanaya E.V., Lukyanov K.V., Zhabitskaya E.I., and Zhabitsky M.V. Modelling of a microscopic optical pion-nucleus potential at energies in the (3,3)-resonance region and nuclear-matter effect on the pion-nucleon amplitude. Preprint JINR, P4-2012-105, Dubna (2012); Physics of Atomic Nuclei, 77 (2014) no.1, 100

[6] Shalaby A.S., Hassan M.Y.M., El-Gogary M.M.H. Nucleus-nucleus scattering in the high-energy approximation and optical folding potential. Brazilian J.Phys., 37 (2007) no.2A, 388

[7] Glauber R.J. High-energy collision theory. in Lectures in Theoretical Physics. Interscience, New York (1959) 315

[8] Satchler R.G. Direct Nuclear Reactions, Clarendon press, Oxford, New York (1983)

[9] Kunz P.D., Rost E. Computational Nuclear Physics, V.2, Eds. Langanke K. et al. Springer-Verlag (1993) 88

[10] Lukyanov V.K., Zemlyanaya E.V., Słowiǹski B.S. Nucleus-nucleus reaction cross-sections calculated for realistic nuclear matter distributions within the Glauber-Sitenko approach. Phys. Atom. Nucl. 67 (2004) 1282

[11] Khoa D.T., Satchler G.R. Generalized folding model for elastic and inelastic nucleus-nucleus scattering using realistic density dependent nucleon-nucleon interaction. Nucl. Phys. A 668 (2000) 3

[12] Patterson J.D., Peterson R.J. Empirical distributions of protons within nuclei. Nucl. Phys. A 717 (2003) 235

[13] Preedom B.M., et. al. A systematic study of $\pi^{+}$and $\pi^{-}$inelastic scattering from ${ }^{28}$ Si in the region of the $\pi N N(3,3)$ resonance. Nucl. Phys. A 326 (1979) 385

[14] Gretillat P., et. al. Study of $\pi^{+}$and $\pi^{-}$elastic scattering from ${ }^{40} \mathrm{Ca}$ and ${ }^{48} \mathrm{Ca}$ in the region of the $\pi N(3,3)$ resonance. Nucl. Phys. A 364 (1981) 270

[15] Olmer C., et. al. Elastic and inelastic scattering of $162 \mathrm{MeV}$ pions on ${ }^{28} \mathrm{Si},{ }^{58} \mathrm{Ni}$, and ${ }^{208} \mathrm{~Pb}$. Phys. Rev. C 21 (1980) 254

[16] Geesaman D.F., Olmer C., Zeidman B., et. al. Elastic and inelastic scattering of 291-Mev pions by ${ }^{9} \mathrm{Be}, \mathrm{Si},{ }^{58} \mathrm{Ni}$, and ${ }^{208} \mathrm{~Pb}$. Phys. Rev. C 23 (1981) 2635

[17] Locher M.P., Steinmann O., Straumann N. Why is the 33-resonance shifted in nuclei? Nucl. Phys. B 27 (1971) 598 